\title{
Short communication: Evidence for a major gene by polygene interaction for milk production traits in German Holstein dairy cattle
}

\author{
M. Streit, ${ }^{*}$ N. Neugebauer, ${ }^{*}$ T. H. E. Meuwissen, $†$ and J. Bennewitz ${ }^{\star 1}$ \\ *Institute of Animal Husbandry and Breeding, University of Hohenheim, 70599 Stuttgart, Germany \\ †Department of Animal and Aquacultural Sciences, Norwegian University of Life Sciences, Ås, Norway
}

\begin{abstract}
The present study investigated putative interaction effects between the DGAT1 K232A mutation and the polygenic term (i.e., all genes except $D G A T 1$ ) for 5 milk production traits in the German Holstein dairy cattle population. Mixed models were used, and the test for interaction relied on the comparison of polygenic variance components depending on the sire's genotypes at DGAT1 K232A. Substitution effects were highly significant for all traits. Significant interaction effects were found for milk fat and protein percentage.

Key words: DGAT1, epistasis, dairy cattle
\end{abstract}

\section{Short Communication}

The genetic variation of quantitative traits is due to polymorphic loci with additive and nonadditive genetic effects (Falconer and Mackay, 1996). Nonadditive genetic effects usually comprise interactions between alleles at the same loci (dominance) or at different loci (epistasis). In outbred populations, most of the genetic variance is additive, which is mainly due to the U-shaped distribution of gene frequency (Hill et al., 2008). On the gene or QTL level, epistatic effects were frequently found in experimental crosses (Carlborg and Haley, 2004). They occasionally explained a substantial proportion of the genetic variance, which is also due to the intermediate gene frequencies in these crosses. In outbred livestock populations (e.g., dairy cattle), however, almost no attempts were made to map epistatic effects between QTL, which is most likely due to restricted experimental power. However, recently Hinrichs et al. (2010) reported interaction effects between a major gene (i.e., DGAT1 K232A) and QTL on BTA5 and BTA14 for fat and protein percentage in a dairy cattle population. The DGAT1 gene is known to affect milk production traits in dairy cattle (Grisart et al., 2002; Winter et al., 2002). The DGAT1 gene encodes

Received September 17, 2010.

Accepted November 2, 2010.

${ }^{1}$ Corresponding author: j.bennewitz@uni-hohenheim.de an enzyme that catalyzes the reaction of diacylglycerol and fatty acyl coenzyme A to form triglycerides. Both studies found a nonconservative substitution of lysine by alanine (K232A) in DGAT1 caused by an adenine/ adenine to guanine/cytosine dinucleotide substitution. Effects of DGAT1 K232A in the German Holstein dairy cattle population were estimated by Thaller et al. (2003) and Bennewitz et al. (2004). Both authors found a strong allele substitution effect for milk production traits. The lysine variant increased fat yield and percentage, as well as protein percentage; the alanine variant increased milk and protein yield.

The aim of the present study was to investigate interaction effects between the DGAT1 K232A mutation and the polygenic component (i.e., all other genes) for milk production traits in German Holsteins.

The pedigree contained 1,153 progeny-tested and DGAT1 K232A-genotyped German Holstein sires. Data from the first lactation for the following 5 traits were considered: milk yield, fat yield, protein yield, fat percentage, and protein percentage. For the yield traits, daughter yield deviations (DYD) were used, which were multiplied by 2 . For the percentage traits, no DYD were available; therefore, EBV were used. The EBV were not de-regressed, because they showed a high reliability. The DYD and EBV were taken from the routine national sire evaluation from 2009. For a summary statistic see Table 1.

Two statistical models were applied. The first one was

$$
\mathbf{y}=\mathbf{X b}+\mathbf{Z u}+\mathbf{e},
$$

where $\mathbf{y}$ is the vector of phenotypes and $\mathbf{b}$ is a $2 \times 1$ vector containing a fixed mean effect and a fixed regression coefficient on the number of lysine alleles $(0,1$, or 2 ) of the animals at DGAT1 K232A. The regression coefficient in $\mathbf{b}$ represents the average allele substitution effect; $\mathbf{X}$ is the corresponding incidence matrix, $\mathbf{u}$ is the vector of polygenic effects with $\mathbf{Z}$ as the corresponding design matrix, and $\mathbf{e}$ is a vector of residuals. The expectation $(E)$ of $\mathbf{y}$ is $E(\mathbf{y})=\mathbf{X} \mathbf{b}$ and the variance (var) is $\operatorname{var}(\mathbf{y})=\mathbf{Z} \mathbf{A} \sigma_{u}^{2} \mathbf{Z}^{\prime}+\mathbf{I} \sigma_{e}^{2}$, where $\mathbf{A}$ is the 
Table 1. Summary statistics of the dependent variables $(\mathrm{n}=1,153)$

\begin{tabular}{llrrrr}
\hline Trait & Unit & Mean & \multicolumn{1}{c}{ SD } & Minimum & Maximum \\
\hline Fat $(\mathrm{kg})$ & DYD $^{1}$ & 4.71 & 20.08 & -56.19 & 79.05 \\
Fat $(\%)$ & EBV & -0.01 & 0.27 & -0.84 & 0.99 \\
Protein $(\mathrm{kg})$ & DYD & 4.01 & 16.76 & -47.77 & 54.68 \\
Protein (\%) & EBV & -0.01 & 0.11 & -0.39 & 0.43 \\
Milk $(\mathrm{kg})$ & DYD & 156.72 & 574.78 & $-1,571.88$ & $2,192.88$ \\
\hline
\end{tabular}

${ }^{1}$ Daughter yield deviations.

numerator relationship matrix among the animals, $\mathbf{Z}^{\prime}$ is the transpose of $\mathbf{Z}, \sigma_{u}^{2}$ is the polygenic variance, $\mathbf{I}$ is an identity matrix, and $\sigma_{e}^{2}$ the residual variance.

In the second model, the polygenic term (i.e., the genetic term corrected for the effect of DGAT1 K232A mutation; $\mathbf{u}$ in model [1]) was split into a polygametic term of gametes associated with the lysine $\left(\mathbf{u}_{L}\right)$ and with the alanine $\left(\mathbf{u}_{A}\right)$ variant at DGAT1 K232A. For sires being homozygous for lysine (alanine) at DGAT1 K232A, the polygametic lysine (alanine) variant affected the phenotype 2 times, for heterozygous sires each polygametic term affected the phenotype one time. Subsequently, the model was

$$
\mathbf{y}=\mathbf{X b}+\mathbf{Z}_{1} \mathbf{u}_{L}+\mathbf{Z}_{2} \mathbf{u}_{A}+\mathbf{e},
$$

where $\mathbf{Z}_{1}$ and $\mathbf{Z}_{2}$ are design matrices linking the phenotypes to the corresponding polygametic effects. Matrix $\mathbf{Z}_{1}\left(\mathbf{Z}_{2}\right)$ contains a 0,1 , or 2 if the sire carries 0,1 , or 2 copies of the lysine (alanine) alleles at DGAT1 K232A, respectively. The remaining terms are as defined above. The expectation of $\mathbf{y}$ is again $E(\mathbf{y})=\mathbf{X b}$, and the variance is

$$
\operatorname{var}(\mathbf{y})=\left[\begin{array}{ll}
\mathbf{Z}_{1} & \mathbf{Z}_{2}
\end{array}\right]\left[\begin{array}{cc}
\mathbf{A} \sigma_{u_{L}}^{2} & \mathbf{A} \sigma_{u_{L}, u_{A}} \\
\mathbf{A} \sigma_{u_{L}, u_{A}} & \mathbf{A} \sigma_{u_{A}}^{2}
\end{array}\right]\left[\begin{array}{l}
\mathbf{Z}_{1}^{\prime} \\
\mathbf{Z}_{2}^{\prime}
\end{array}\right]+\mathbf{I} \boldsymbol{\sigma}_{e}^{2}
$$

where $\sigma_{u_{L}}^{2}$ is the lysine polygametic variance, $\sigma_{u_{A}}^{2}$ is the alanine polygametic variance, and $\sigma_{u_{L}, u_{A}}$ is the covariance between lysine and alanine variants. Parameters of both models were estimated using ASReml (Gilmour et al., 2006). The REML log-likelihood of model [1] and [2] was calculated and was denoted as $\log l_{m 1}$ and $\log l_{m 2}$, respectively.

For the test of putative DGAT1 K232A by polygene interaction effects, the null hypothesis was $H_{0}: \sigma_{u_{L}}^{2}=\sigma_{u_{A}}^{2}=\sigma_{u_{L}, u_{A}}$. The alternative hypothesis was $H_{1}: \sigma_{u_{L}}^{2} \neq \sigma_{u_{A}}^{2}$ or $\sigma_{u_{L}}^{2}=\sigma_{u_{A}}^{2} \neq \sigma_{u_{L}, u_{A}}$. Under the assumption of the null hypothesis, all 3 variance components in model [2] are the same and $\sigma_{u_{L}}^{2}+\sigma_{u_{A}}^{2}+2 \times \sigma_{u_{L}, u_{A}}=\sigma_{u}^{2}$ from model [1]. In this case, the expectations and variances of the observations are the same for each individual, and, hence, both models are equivalent. Therefore, the $\log l_{m 1}$ is the log-likelihood under the null hypothesis. This leads to the restricted likelihood ratio test $(R L R T)$ with $R L R T=2\left(\log l_{m 2}-\log l_{m 1}\right)$. With the assumption of between-subject independence, the asymptotic distribution of the RLRT under the null hypothesis follows a mixture of $2 \chi^{2}$-distributions with 1 and 2 degrees of freedom (Self and Liang, 1987). Because the animals are related, and hence the assumption of between-subject independence is not fulfilled, we used a conservative test with a $\chi^{2}$-distribution with 2 degrees of freedom. The resulting comparisonwise error probability was denoted as $p_{c}$. Five tests were conducted, one for each trait. The correction for the resulting multiple testing was done using the Bonferroni correction, resulting in experiment-wise error probabilities $\left(p_{e}\right)$, $p_{e}=1-\left(1-p_{c}\right)^{5}$.

The results of model [1] are presented in Table 2 . The average substitution effect of the lysine variant is positive for fat yield and the 2 percentage traits, and negative for protein and milk yield. These estimates are in agreement with those of Thaller et al. (2003) and Bennewitz et al. (2004) and also with those estimated in the French Holstein breed (Gautier et al., 2007). Allele substitution effects were all highly significant $(P<$ 0.001). Results of model [2] and of the test for interaction effects are shown in Table 3. Experimentwise significant interaction effects were found for fat percentage $\left(p_{e}<0.05\right)$. Additional comparisonwise interaction effects were found for protein percentage $\left(p_{c}<0.05\right)$. These results agree with the interaction effects detected by Hinrichs et al. (2010). An additional weak, but not significant, interaction was found for fat yield. For these traits, the polygametic alanine variance was between 37 and $41 \%$ higher compared with that of the lysine variant (Table 3). Hence, the DGAT1 K232A lysine variant increased the mean of the phenotypes (Table 2) but 
Table 2. Average DGAT1 K232A lysine allele substitution effect $(\hat{\alpha})$ and polygenic variance component $\left(\hat{\sigma}_{u}^{2}\right)$ results from the model with one polygenic effect (SE in parentheses)

\begin{tabular}{lcc}
\hline Trait & \multicolumn{1}{c}{$\hat{\alpha}$} & $\hat{\sigma}_{u}^{2}$ \\
\hline Fat $(\mathrm{kg})$ & $7.668(0.857)$ & $305.207(39.844)$ \\
Fat $(\%)$ & $0.281(0.009)$ & $416.567^{1}(42.205)$ \\
Protein $(\mathrm{kg})$ & $-6.508(0.714)$ & $209.009(22.843)$ \\
Protein $(\%)$ & $0.059(0.005)$ & $114.790^{1}(9.250)$ \\
Milk $(\mathrm{kg})$ & $-302(23.741)$ & $256,367(29,672)$ \\
\hline
\end{tabular}

${ }^{1}$ Multiplied by 1,000 .

decreased the polygametic variance. The estimated correlation between $\mathbf{u}_{L}$ and $\mathbf{u}_{A}$ was $\hat{r}<1$ for fat yield and especially fat percentage. This is not the case for protein percentage $(\hat{r} \approx 0.99)$, which implies that the improved fit of model [2] is due to heterogeneous variances (i.e., $u_{A}=c \times u_{L}$, where $\mathrm{c}$ is a constant and equal to 0.84 in this case, not shown elsewhere). One possible explanation for the detected interaction effects might be the relationship between the enzyme activity and amount of end product (i.e., the amount of milk, fat yield, fat percentage, protein yield, and protein percentage). The DGAT1 catalyzes the final step of the triglyceride synthesis. Grisart et al. (2004) found a higher enzyme activity level in producing triglycerides for the DGAT1 K232A lysine variant. Kacser and Burns (1973) developed a metabolic control theory, which modeled the phenotype as an end product of enzyme activity. The enzyme activity causes a flux through metabolic pathways with a hyperbolic relationship. At a low flux level, an infinitesimal change of the enzyme activity results in a comparable larger change of the flux, and hence, of the enzyme end product (the milk traits in our study). The opposite holds, if the flux level is already at a high level. Kacser and Burns (1981) used this theory to derive some conclusions for the dominance phenomenon, but it might also be valid in this case to explain the detected interaction effects. The limiting factor for a higher triglyceride synthesis might be the limited availability of the 2 substrates diacylglycerol and fatty acyl coenzyme A when forming triglycerides. If animals carry the favorable lysine alleles, the effect of additional favorable alleles at other genes might be reduced, because they have to compete for the 2 substrates.

Intuitively, one might argue that it is not possible with these data to test for interaction effects. Genotypes and phenotypes are not recorded within the same generation and gene combinations break down during meiosis. However, the DGAT1 K232A frequencies among daughters of DGAT1 K232A-homozygous sires and DGAT1 K232A-heterozygous sires differ by onequarter, and among daughters of alternative DGAT1 K232A-homozygous sires by one-half. These daughters largely determine the DYD and EBV of the sires used in the study. With respect to this, it would have been better to estimate the DYD and EBV using a sire model and ignore the relationships between the sires as done by Seidenspinner et al. (2009). However, this was not possible in this study. In theory, interaction between genes can be classified as additive $\times$ additive or additive $\times$ dominance interactions, among others (Falconer and Mackay, 1996). The identified interaction terms are most likely due to an interaction between the additive DGAT1 K232A effect and the additive polygenic effect, because the phenotypes used (DYD and EBV) should contain a part of the additive $\times$ additive interaction (Falconer and Mackay, 1996, p. 154). It may be noted that it is not possible with this data set to detect interaction effects within DGAT1 K232A (dominance), because the daughters only inherit the additive effect of the sires, but not their dominance effect.

Theoretically, the significant interactions could be used in breeding schemes. For each sire, 3 polygenic breeding values can be calculated, depending on the

Table 3. Estimated polygametic variance and covariance components $\left(\hat{\sigma}_{u_{L}}^{2}, \hat{\sigma}_{u_{A}}^{2}, \hat{\sigma}_{u_{L}, u_{A}}\right)$, estimated correlation between the two polygametic terms $(\hat{r}$; results from the model with 2 polygametic effects), and restricted likelihood ratio test statistics $(R L R T)$ with comparisonwise and experimentwise error probability $\left(p_{c} \text { and } p_{e} \text {, respectively }\right)^{1}$

\begin{tabular}{lcccccc}
\hline Trait & $\hat{\sigma}_{u_{L}}^{2}$ & $\hat{\sigma}_{u_{A}}^{2}$ & $\hat{\sigma}_{u_{L} u_{A}}$ & $\hat{r}$ & $R L R T$ & $p_{c}$ \\
\hline Fat $(\mathrm{kg})$ & $70.14(10.60)$ & $95.81(14.07)$ & $74.23(11.37)$ & 0.906 & 5.24 & 0.073 \\
Fat (\%) & $94.42^{2}(12.01)$ & $133.00^{2}(16.80)$ & $87.06^{2}(14.51)$ & 0.777 & 10.94 & 0.004 \\
Protein (kg) & $51.24(2.14)$ & $57.09(2.38)$ & $54.05(2.25)$ & 0.999 & NS & NS \\
Protein (\%) & $24.94^{2}(2.52)$ & $35.28^{2}(3.76)$ & $29.29^{2}(2.86)$ & 0.988 & 6.98 & 0.031 \\
Milk (kg) & $64,460(2,687)$ & $63,770(2,658)$ & $60,300(2,513)$ & 0.941 & NS & NS \\
\hline
\end{tabular}

${ }^{1}$ Standard errors are given in parentheses; $\hat{\sigma}_{u_{L}}^{2}=$ lysine polygametic variance; $\hat{\sigma}_{u_{A}}^{2}=$ alanine polygametic variance; $\hat{\sigma}_{u_{L}, u_{A}}=$ covariance between $u_{L}$ and $u_{A}$.

${ }^{2}$ Multiplied by 1,000 . 
DGAT1 K232A genotypes of the sample in which the sire will be used. In a homozygous lysine (alanine) sample, the polygenic EBV would be $2 \times \hat{u}_{L}\left(2 \times \hat{u}_{A}\right)$ and in a heterozygous sample it would be $\hat{u}_{L}+\hat{u}_{A}$. However, because the DGAT1 K232A genotypes of the samples are often unknown and usually heterogeneous, this is not a practical approach. Additionally, rank correlations between these 3 polygenic EBV were always above 0.95 (data not shown), suggesting very little reranking of the sires.

\section{ACKNOWLEDGMENTS}

The authors thank the Vereinigte Informationssysteme für Tierhaltung w.V. (VIT), Verden, Germany for providing the data. The study was conducted within the FUGATO-plus project GeneDialog. Partial support for M. Streit was through a grant from the Deutsche Forschungsgemeinschaft, DFG.

\section{REFERENCES}

Bennewitz, J., N. Reinsch, S. Paul, C. Looft, B. Kaupe, C. Weimann, G. Erhardt, G. Thaller, Ch. Kühn, M. Schwerin, H. Thomsen, F. Reinhardt, R. Reents, and E. Kalm. 2004. The DGAT1 K232A mutation is not solely responsible for the milk production quantitative trait locus on the bovine chromosome 14. J. Dairy Sci. $87: 431-442$.

Carlborg, O., and C. S. Haley. 2004. Epistasis: Too often neglected in complex trait studies? Genetics 5:618-625.

Falconer, D. S., and T. F. C. Mackay. 1996. Introduction to quantitative genetics. 4th ed. Longman Scientific and Technical, New York, NY.

Gautier, M., A. Capitan, S. Fritz, A. Eggen, D. Boichard, and T. Druet. 2007. Characterization of the DGAT1 K232A and variable number of tandem repeat polymorphisms in French dairy cattle. J. Dairy Sci. 90:2980-2988.

Gilmour, A. R., B. J. Gogel, B. R. Cullis, and R. Thompson. 2006 ASReml User Guide 2.0. VSN International Ltd., Hemel Hempstead, UK.

Grisart, B., W. Coppieters, F. Farnir, L. Karim, C. Ford, P. Berzi, N. Cambisano, M. Mni, S. Reid, P. Simon, R. Spelman, M. Georges, and R. Snell.2002. Positional candidate cloning of a QTL in dairy cattle: Identification of a missense mutation in the bovine DGAT1 K232A gene with major effect on milk yield and composition. Genome Res. 12:222-231.

Grisart, B., F. Farnir, L. Karim, N. Cambisano, J.-J. Kim, A. Kvasz, M. Mni, P. Simon, J.-M. Frère, W. Coppieters, and M. Georges. 2004. Genetic and functional confirmation of the causality of the DGAT1 K232A quantitative trait nucleotide in affecting milk yield and confirmation. Proc. Natl. Acad. Sci. USA 101:2398-2403.

Hill, W. G., M. E. Goddard, and P. M. Visscher. 2008. Data and theory point to mainly additive genetic variance for complex traits. PLoS Genet. 4:e1000008 doi:10.1371/journal.pgen.1000008.

Hinrichs, D., J. Bennewitz, J. Tetens, N. Reinsch, and G. Thaller. 2010. A two step approach to detect interaction between QTL and a known major gene in dairy cattle. Communication No. 379, 9th World Congress on Genetics Applied to Livestock Production, Leipzig, Germany.

Kacser, H., and J. A. Burns. 1973. The control of flux. Symp. Soc. Exp. Biol. 27:65-104.

Kacser, H., and J. A. Burns. 1981. The molecular basis of dominance. Genetics 97:639-666.

Seidenspinner, T., J. Bennewitz, F. Reinhardt, and G. Thaller. 2009 The need for sharp phenotypes in QTL mapping for calving traits in dairy cattle. J. Anim. Breed. Genet. 126:455-462.

Self, S. G., and K.-Y. Liang. 1987. Asymptotic properties of maximum likelihood estimators and likelihood ratio tests under nonstandard conditions. J. Am. Stat. Assoc. 82:605-610.

Thaller, G., W. Krämer, A. Winter, B. Kaupe, G. Erhardt, and R. Fries. 2003. Effects of DGAT1 K232A variants on milk production traits in German cattle breeds. J. Anim. Sci. 81:1911-1918.

Winter, A., W. Krämer, F. A. O. Werner, S. Kollers, S. Kata, G. Durstewitz, J. Buitkamp, J. E. Womack, G. Thaller, and R. Fries. 2002. Association of a lysine-232/alanine polymorphism in a bovine gene encoding acyl-CoA:diacylglycerol acyltransferase (DGAT1) with variation at a quantitative trait locus for milk fat percentage. Proc. Natl. Acad. Sci. USA 99:9300-9305. 